\title{
On the Efficient Simulation of Outage Probability in a Log-normal Fading Environment
}

\author{
Nadhir Ben Rached, Abla Kammoun, Mohamed-Slim Alouini, and Raul Tempone
}

\begin{abstract}
The outage probability (OP) of the signal-to-interference-plus-noise ratio (SINR) is an important metric that is used to evaluate the performance of wireless systems. One difficulty toward assessing the $\mathrm{OP}$ is that, in realistic scenarios, closed-form expressions cannot be derived. This is for instance the case of the Log-normal environment, in which evaluating the OP of the SINR amounts to computing the probability that a sum of correlated Log-normal variates exceeds a given threshold. Since such a probability does not admit a closed-form expression, it has thus far been evaluated by several approximation techniques, the accuracies of which are not guaranteed in the region of small OPs. For these regions, simulation techniques based on variance reduction algorithms is a good alternative, being quick and highly accurate for estimating rare event probabilities. This constitutes the major motivation behind our work. More specifically, we propose a generalized hybrid importance sampling scheme, based on a combination of a mean shifting and a covariance matrix scaling, to evaluate the OP of the SINR in a Log-normal environment. We further our analysis by providing a detailed study of two particular cases. Finally, the performance of these techniques is performed both theoretically and through various simulation results.
\end{abstract}

\section{Index Terms}

The authors are in Computer, Electrical and Mathematical Science and Engineering (CEMSE) Division, King Abdullah University of Science and Technology (KAUST), Thuwal, Makkah Province, Saudi Arabia , and also members of the KAUST Strategic Research Initiative on Uncertainty Quantification in Science and Engineering (SRI-UQ) (e-mail: nadhir.benrached, abla.kammoun, slim.alouini, raul.tempone@kaust.edu.sa).

Part of this work has been accepted at the IEEE Global Communications Conference (GLOBECOM'2015), San Diego, CA , USA, Dec. 2015 [1]. 
outage probability, sum of correlated Log-normal, importance sampling, mean shifting, covariance matrix scaling.

\section{INTRODUCTION}

The outage probability (OP) of the signal-to-interference-plus-noise ratio (SINR), defined as the probability that the SINR falls bellow a certain threshold, is an important performance metric for wireless communication systems operating over fading channels. Evaluating the OP, while being of major practical interest, is not an easy task, the main difficulty being in the handling of the distribution of the propagation channels. Several distributions can be assumed, among which we distinguish the Log-normal distribution which is shown to exhibit a good fit to realistic propagation channels. It has thus far been used to model several types of attenuation including shadowing [2], large-scale fading in the ultra-wideband communications [3], and the weakto-moderate turbulence channels in free-space optical communications [4]. If the Log-normal modeling is considered, the computation of the OP with co-channel interference turns out to be equivalent to the problem of evaluating the probability that a sum of correlated Log-normal random variables (RVs) exceeds a given threshold [5].

Several works have attempted to analyze this problem but, to the best of our knowledge, none has successfully provided a closed-form expression for its probability density function (PDF). Failing that, several researchers resorted to different approximation techniques of the sum distribution of correlated Log-normal RVs. The methods presented in the literature can be mainly classified into two categories. The first category includes methods where the Log-normal sum is approximated by another Log-normal RV. These are essentially the methods in [6] and in [7] wherein the parameters of the approximate Log-normal RV are computed using moment matching. In the same vein, moment matching in the logarithm domain and moment generating function based approaches have been derived to determine the parameters of the approximating distributions in [8] and [9], respectively. The authors in [5] have further extended the moment matching approach [6] to problems involving binary weighted sums of correlated Log-normal variates, by incorporating second order statistics. Interested readers are referred to [10] and the references therein for a more comprehensive list. A second class of techniques employing non Log-normal approximation densities has been proposed as well. For instance, a variant of type IV Pearson distribution has been used to approximate the Log-normal sum distribution [10]- 
[12]. In [13], an exponential function in the Log-normal probability scale has been proposed to approximate the cumulative distribution function of the sum of Log-normal variates. Generally, the accuracy of the previously cited approaches and their related works is not guaranteed for all scenarios, i.e their accuracy can degrade for a particular choice of the distribution parameters or for an arbitrary number of summands. This has clearly been illustrated in [14] where the authors have shown that an optimal Log-normal approximation to the sum distribution fails to be accurate in estimating small probabilities. This range of probabilities corresponds to the region of operating OP and is, as such, of major practical interest for many practical systems [15].

On the other hand, it is well-acknowledged that the framework of rare events simulation can represent a good efficient solution, especially when ranges of small OP are considered. While several advances in the development of these methods have recently been recorded, their popularity among researchers in wireless communication systems has thus far remained limited. Surprisingly, after forerunning work in the 1980s and 1990s [16], [17] and references therein, application of rare events simulation methods for the purpose of assessing the performance of wireless communications systems has taken a back seat until recently [18]-[20], although their particular adequacy to the estimation of small probabilities make them all the more suitable to the estimation of operating OP. This lies behind the major contribution of our work. In particular, we propose a generalized hybrid importance sampling (IS) scheme to efficiently estimate the $\mathrm{OP}$ in the presence of noise and interference in a Log-normal environment. The proposed method belongs to the class of IS-based approaches, which basically consist in changing the sampling distribution to a new one satisfying better properties. In our situation, we propose to select the new sampling distribution by jointly shifting the mean and scaling the covariance matrix corresponding to the original multivariate normal distribution. Two interesting particular cases of the generalized hybrid IS approach are studied in detail in this paper. The first is only based on scaling the covariance matrix which finds its root in [21] and was the subject of the conference version [1]. The second particular IS scheme is constructed by considering a particular structure between the shifting and the scaling parameters. We present a profound analysis of all these approaches. Particularly, we show that they all satisfy the asymptotic optimality criterion, a property that is well-used to measure the efficiency of IS schemes [22]. For the sake of comparison, we study their performances in terms of the coefficient of variation. As an interesting outcome of this analysis, we distinguish the regions wherein only scaling the covariance matrix 
outperforms the hybrid approach and vice versa. It is important to note that contrary to previous works that apply IS techniques for the purpose of analyzing performances [23], our work is the first one to take into account the co-channel interference. It constitutes, in this respect, a first step in promoting the use of IS techniques for evaluating the performance of wireless communication systems when operating in realistic scenarios.

The rest of the paper is organized as follows. In Section II, the system model is described. We review in Section III the basic concept of IS. In section IV, the generalized hybrid IS scheme is presented. Moreover, we provide a detailed analysis of two particular scenarios. Finally, simulations are carried out in section V comparing the computational efficiency of the IS schemes with the naive Monte Carlo (MC) approach.

\section{System Model}

In a wireless system with $N$ co-channel interferers, the instantaneous SINR at the desired receiver can be expressed as:

$$
\mathrm{SINR}=\frac{X_{0}}{\sum_{i=1}^{N} X_{i}+\eta}
$$

where $\eta$ is the variance of the additive white Gaussian noise, $X_{0}$ is the received power of the useful signal, $\left\{X_{i}\right\}_{i=1}^{N}$ is the set of received powers of the $N$ interfering signals. The RVs $\left\{X_{i}\right\}_{i=0}^{N}$ are assumed to be independent and not necessarily identically distributed Log-normal variates. A widely used performance measure of wireless communication systems operating over fading channels in the presence of interference is the OP which is defined as the probability that the SINR falls bellow a given threshold $\gamma_{t h}$ :

$$
\alpha=P\left(\operatorname{SINR}<\gamma_{t h}\right)
$$

Let $Y_{i}=\log \left(X_{i}\right)$ be the associated Gaussian RVs with mean $\mu_{i}$ and standard deviation $\sigma_{i}$, $i=0,1, \ldots, N$. The quantity $\alpha$ can then be rewritten as

$$
\alpha=P\left(\sum_{i=0}^{N} \exp \left(Z_{i}\right)>1 / \gamma_{t h}\right)
$$

where

$$
Z_{i}= \begin{cases}Y_{i}-Y_{0} & i=1,2, \ldots, N \\ \log (\eta)-Y_{0} & i=0 .\end{cases}
$$


Hence, we transform the problem of evaluating the OP to that of computing the probability that a sum of correlated Log-normal RVs exceeds a certain threshold, i.e. the probability of the event $\left\{\sum_{i=0}^{N} \exp \left(Z_{i}\right)>1 / \gamma_{t h}\right\}$, where vector $\mathbf{Z}=\left(Z_{0}, Z_{1}, \ldots, Z_{N}\right)^{t}$ has a joint $N+1$-dimensional Gaussian distribution $\mathcal{N}(\boldsymbol{\mu}, \boldsymbol{\Sigma})$ with PDF

$$
f(\boldsymbol{z})=\frac{\exp \left(-\frac{1}{2}(\boldsymbol{z}-\boldsymbol{\mu})^{t} \boldsymbol{\Sigma}^{-\mathbf{1}}(\boldsymbol{z}-\boldsymbol{\mu})\right)}{\sqrt{\operatorname{det} \boldsymbol{\Sigma}(2 \pi)^{N+1}}}
$$

$\boldsymbol{\mu}$ being its mean vector

$$
\boldsymbol{\mu}=\left(\log (\eta)-\mu_{0}, \mu_{1}-\mu_{0}, \mu_{2}-\mu_{0}, \ldots, \mu_{N}-\mu_{0}\right)^{t}
$$

and $\Sigma$ its covariance matrix given by

$$
\boldsymbol{\Sigma}=\sigma_{0}^{2} \boldsymbol{a} \boldsymbol{a}^{t}+\operatorname{diag}\left[\left(0, \sigma_{1}^{2}, \ldots, \sigma_{N}^{2}\right)^{t}\right]
$$

with $\boldsymbol{a}$ being the vector of all ones and $\operatorname{diag}[\boldsymbol{x}]$ denoting the diagonal matrix whose diagonal elements are the entries of $\boldsymbol{x}$.

\section{IMPORTANCE SAMPLING}

Naive MC simulation is the standard technique to estimate $\alpha$ by

$$
\hat{\alpha}_{M C}=\frac{1}{M} \sum_{i=1}^{M} H\left(\sum_{j=0}^{N} \exp \left(Z_{j}^{(i)}\right)\right)
$$

where $\left\{\left(Z_{0}^{(i)}, Z_{1}^{(i)}, \ldots, Z_{N}^{(i)}\right)^{t}\right\}_{i=1}^{M}$ are independent realizations of the random vector $\mathbf{Z}$ under the PDF $f$ and $H(X)$ is the indicator function corresponding to the set $\left\{X>1 / \gamma_{t h}\right\}$. Since for typical wireless communication systems, operating OP take relatively small values, the use of naive MC simulation will inevitably require high computational complexity and thus does not constitute a good option. Variance reduction algorithms represent, in this respect, good alternatives that achieve high computational gains over naive MC simulations. This motivates us to consider in the present work the use of IS-based simulation approaches for the estimation of the probability of interest $\alpha$ [24].

Prior to delving into the core of the present work, we shall first, for the reader convenience, review the concept of IS. The methodology behind IS is to perform a change of the probability measure with the aim of achieving a variance reduction. More clearly, instead of sampling 
according to $f$, the idea of IS is to consider a new PDF $g$ according to which sampling is performed. This can be done by rewriting $\alpha$ as follows

$$
\begin{aligned}
\alpha & =\mathbb{E}_{f}\left[H\left(\sum_{i=0}^{N} \exp \left(Z_{i}\right)\right)\right] \\
& =\int H\left(\sum_{i=0}^{N} \exp \left(z_{i}\right)\right) f\left(z_{0}, \ldots, z_{N}\right) d z_{0} \ldots d z_{N} \\
& =\int H\left(\sum_{i=0}^{N} \exp \left(z_{i}\right)\right) \frac{f\left(z_{0}, \ldots, z_{N}\right)}{g\left(z_{0}, \ldots, z_{N}\right)} g\left(z_{0}, \ldots, z_{N}\right) d z_{0} \ldots d z_{N} \\
& =\mathbb{E}_{g}\left[H\left(\sum_{i=0}^{N} \exp \left(Z_{i}\right)\right) L\left(Z_{0}, \ldots, Z_{N}\right)\right]
\end{aligned}
$$

where $\mathbb{E}_{f}[\cdot]$ and $\mathbb{E}_{g}[\cdot]$ are the expectations under the PDFs $f$ and $g$ respectively, whereas $L\left(Z_{0}, \ldots, Z_{N}\right)$ is the likelihood ratio defined as:

$$
L\left(Z_{0}, \ldots, Z_{N}\right)=\frac{f\left(Z_{0}, \ldots, Z_{N}\right)}{g\left(Z_{0}, \ldots, Z_{N}\right)} .
$$

The IS estimator is thus defined as:

$$
\hat{\alpha}_{I S}=\frac{1}{M} \sum_{i=1}^{M} T_{\gamma_{t h}}\left(\mathbf{Z}^{(i)}\right)
$$

where $\left\{\mathbf{Z}^{(i)}=\left(Z_{0}^{(i)}, Z_{1}^{(i)}, \ldots, Z_{N}^{(i)}\right)^{t}\right\}_{i=1}^{M}$ are independent realizations of the random vector $\mathbf{Z}$ under the PDF $g$ and $T_{\gamma_{t h}}(\mathbf{Z})$ is given by:

$$
T_{\gamma_{t h}}(\mathbf{Z})=H\left(\sum_{i=0}^{N} \exp \left(Z_{i}\right)\right) L\left(Z_{0}, \ldots, Z_{N}\right) .
$$

The crucial step of IS lies in the choice of an appropriate PDF $g$ that results in a substantial variance reduction. In fact, a good choice of the PDF $g$ should emphasize the generation of important samples, i.e. samples belonging to the set $\left\{\sum_{i=0}^{N} \exp \left(Z_{i}\right)>1 / \gamma_{t h}\right\}$ for a sufficiently small threshold $\gamma_{t h}$. Ensuring that these important realizations are frequently sampled is likely to lead to a reduction in the variance of the IS estimator ${ }^{1}$.

To measure the goodness of an IS scheme, many performance measures have been used in the literature [22]. Among the most used metrics, we distinguish the asymptotic optimality which

\footnotetext{
${ }^{1} \mathrm{~A}$ reduction in the variance of the IS estimator can be guaranteed if the boundaries of the event are known. However, most often the boundaries of the event are not known, see [25], [26].
} 
holds when:

$$
\lim _{\gamma_{t h} \rightarrow 0} \frac{\log \left(\mathbb{E}_{g}\left[T_{\gamma_{t h}}^{2}(\mathbf{Z})\right]\right)}{\log (\alpha)}=2 .
$$

Note that this limit cannot be made larger since:

$$
\mathbb{E}_{g}\left[T_{\gamma_{t h}}^{2}(\mathbf{Z})\right] \geq \alpha^{2}
$$

and thus applying the logarithm on both sides and using the fact that $\log (\alpha)<0$, we get:

$$
\frac{\log \left(\mathbb{E}_{g}\left[T_{\gamma_{t h}}^{2}(\mathbf{Z})\right]\right)}{\log (\alpha)} \leq 2 .
$$

The asymptotic optimality reveals two interesting feature. From (12), we easily observe that when $\alpha^{2} \rightarrow 0$ at an exponential rate, the second moment goes also to zero with the same exponential rate. This represents the best rate that the second moment can achieve as per (14). Moreover, it can be easily shown that whenever the asymptotic optimality holds, the number of simulation runs $M$ needed to ensure a fixed accuracy requirement satisfies $M=o\left(\alpha^{-\epsilon}\right)$ for all $\epsilon>0$, see [20]. This has to be compared with the naive MC approach which requires a number of simulation runs of the order of $\alpha^{-1}$ in order to meet the same accuracy requirement.

\section{Generalized HybRID IS SCHEME}

The main reason behind the failure of the naive MC approach inheres in its inability to generate a sufficient number of samples in the set $\left\{\sum_{i=0}^{N} \exp \left(Z_{i}\right)>1 / \gamma_{t h}\right\}$ when $\gamma_{t h}$ is sufficiently small. In order to overcome such a problem, we propose a generalized hybrid IS scheme that consists in both shifting the mean vector $\boldsymbol{\mu}$ by the vector $\theta_{1} \boldsymbol{a}$ and inflating the covariance matrix $\boldsymbol{\Sigma}$ by a factor $\frac{1}{1-\theta_{2}}$, where $\theta_{1} \geq 0$ and $0 \leq \theta_{2}<1$. More precisely, the IS PDF $g$ used in the proposed generalized hybrid approach is a multivariate Gaussian PDF with a shifted mean $\boldsymbol{\mu}+\theta_{1} \boldsymbol{a}$ and a scaled covariance matrix $\Sigma /\left(1-\theta_{2}\right)$ :

$$
\begin{aligned}
g(\boldsymbol{z}) & =\frac{\exp \left(-\frac{1-\theta_{2}}{2}\left(\boldsymbol{z}-\left(\boldsymbol{\mu}++\theta_{1} \boldsymbol{a}\right)\right)^{t} \boldsymbol{\Sigma}^{-\mathbf{1}}\left(\boldsymbol{z}-\left(\boldsymbol{\mu}+\theta_{1} \boldsymbol{a}\right)\right)\right)}{\sqrt{\operatorname{det}\left(\boldsymbol{\Sigma} /\left(1-\theta_{2}\right)(2 \pi)^{N+1}\right.}} \\
& \triangleq g_{\theta_{1}, \theta_{2}}(\boldsymbol{z}) .
\end{aligned}
$$

The Scalars $\theta_{1}$ and $\theta_{2}$ should be selected in such a way that when $\gamma_{t h} \rightarrow 0$ the entries of the new mean vector and covariance matrix go to infinity. In doing so, we encourage the sampling of big values of the RVs $Z_{0}, Z_{1}, \ldots, Z_{N}$, which leads to an increase in the probability of the 
event $\left\{\sum_{i=0}^{N} \exp \left(Z_{i}\right)>1 / \gamma_{t h}\right\}$ under the IS distribution $g_{\theta_{1}, \theta_{2}}$. More precisely, by shifting the mean vector and inflating the covariance matrix, we turn the rare event $\left\{\sum_{i=0}^{N} \exp \left(Z_{i}\right)>1 / \gamma_{t h}\right\}$ into a non-rare one presenting a higher number of occurrences. This is particularly obtained by imposing that $\theta_{1} \rightarrow \infty$ and $\theta_{2} \rightarrow 1$ as $\gamma_{t h} \rightarrow 0$.

The likelihood ratio (10) corresponding to the generalized hybrid IS approach is given through a simple computation as follows:

$$
\begin{aligned}
& L\left(Z_{0}, Z_{1}, \ldots, Z_{N}\right)=\frac{\exp \left(-\frac{\theta_{2}}{2}(\mathbf{Z}-\boldsymbol{\mu})^{t} \boldsymbol{\Sigma}^{-\mathbf{1}}(\mathbf{Z}-\boldsymbol{\mu})\right)}{\left(1-\theta_{2}\right)^{(N+1) / 2}} \\
& \times \exp \left(\frac{\left(1-\theta_{2}\right) \theta_{1}^{2}}{2} \boldsymbol{a}^{t} \boldsymbol{\Sigma}^{-1} \boldsymbol{a}-\left(1-\theta_{2}\right) \theta_{1}(\mathbf{Z}-\boldsymbol{\mu})^{t} \boldsymbol{\Sigma}^{-1} \boldsymbol{a}\right) .
\end{aligned}
$$

The generalized hybrid IS approach defines a whole class of IS schemes associated with different settings of the parameters $\theta_{1}$ and $\theta_{2}$. In particular, the IS obtained by only scaling the covariance matrix is given by setting $\theta_{1}$ to 0 . This method will be referred to as CMS-based approach and a proper way of selecting the parameter $\theta_{1}$ will be provided. When $\theta_{1}$ and $\theta_{2}$ are different from zero, the analysis of the resulting scheme becomes more difficult. For the sake of simplicity, we consider only the case in which $\theta_{1}=\frac{1}{1-\theta_{2}}$. Note that the purpose of this choice is to facilitate the analysis of the generalized hybrid IS approach. The resulting IS scheme will be termed the mean shift- covariance matrix scaling (MS-CMS) IS approach. In the sequel, we establish the asymptotic optimality of the CMS and MS-CMS based techniques and carry out an asymptotic analysis.

Remark 1. It is worth mentioning that setting $\theta_{2}=0$ yields a mean shift IS approach. Such an approach has been recently proposed in [27] to deal with the efficient simulation of the left-tail of the sum of correlated Log-normal RVs, i.e. the probability that a sum of correlated Lognormal RVs falls below a sufficiently small threshold. However, in the present work where we are interested in the efficient simulation of the probability that a sum of correlated Log-normal variates exceeds a sufficiently large threshold, we can easily prove that the mean shift technique is not efficient in the sense that it does not achieve the asymptotic optimality property (12).

To make a comparison between the CMS and MS-CMS based techniques, we propose to derive their asymptotic coefficient of variations. To this end, the following Lemma which provides a closed-form expression for the second moment of the RV $T_{\gamma_{t h}}(\mathbf{Z})$ will be very useful. Note that this Lemma can be used for both schemes as it applies for any given $\theta_{1} \geq 0$ and $0 \leq \theta_{2}<1$. 
Lemma 1. The second moment of $T_{\gamma_{t h}}(\mathbf{Z})$ satisfies:

$$
\begin{aligned}
\mathbb{E}_{g_{\theta_{1}, \theta_{2}}}\left[T_{\gamma_{t h}}^{2}(\mathbf{Z})\right] & =\left(1-\theta_{2}^{2}\right)^{-\frac{N+1}{2}} \exp \left(\frac{\theta_{1}^{2}\left(1-\theta_{2}\right)}{1+\theta_{2}} \boldsymbol{a}^{t} \boldsymbol{\Sigma}^{-1} \boldsymbol{a}\right) \\
& \times P_{\theta_{1}, \theta_{2}}\left(\sum_{i=0}^{N} \exp \left(Z_{i}\right)>\frac{1}{\gamma_{t h}}\right)
\end{aligned}
$$

where $P_{\theta_{1}, \theta_{2}}(\cdot)$ is the probability measure under which the random vector $\mathbf{Z}$ follows a multivariate Normal $\mathcal{N}\left(\boldsymbol{\mu}-\frac{\left(1-\theta_{2}\right) \theta_{1}}{1+\theta_{2}} \boldsymbol{a}, \frac{\boldsymbol{\Sigma}}{1+\theta_{2}}\right)$.

Proof: See Appendix A.

With this Lemma at hand, we are now ready to analyze the performances of CMS and MSCMS based schemes.

\section{A. CMS IS Based Approach}

Herein, we consider the case in which $\theta_{1}=0$ and $\theta_{2}=\theta$. This corresponds to the IS scheme where we only scale the covariance matrix $\Sigma$ by $\frac{1}{1-\theta}$. Its corresponding IS PDF $g_{0, \theta}(\cdot)$ in (15) will be denoted by $g_{\theta}(\cdot)$. Recall that $\theta$ satisfy $0 \leq \theta<1$ and should tend to 1 as $\gamma_{t h}$ goes to zero. From (16), the likelihood ratio associated with the CMS technique is

$$
L\left(Z_{0}, Z_{1}, \ldots, Z_{N}\right)=\frac{\exp \left(-\frac{\theta}{2}(\mathbf{Z}-\boldsymbol{\mu})^{t} \boldsymbol{\Sigma}^{-\mathbf{1}}(\mathbf{Z}-\boldsymbol{\mu})\right)}{(1-\theta)^{(N+1) / 2}} .
$$

From now on, to ease the notations, the RV $T_{\gamma_{t h}}(\mathbf{Z})$ obtained by the CMS approach is denoted by $T_{\gamma_{t h}, C M S, \theta}(\mathbf{Z})$, while its corresponding IS estimator is denoted by $\hat{\alpha}_{I S, C M S, \theta}$. It was shown in [21] that the CMS approach achieves the asymptotic optimality property (12) if $\theta$ is selected in such way that:

$$
(1-\theta)^{-1}=o\left(\exp \left(p \log \left(1 / \gamma_{t h}\right)^{2}\right)\right), \text { for all } p>0 . \gamma_{t h} \rightarrow 0
$$

While the above relation ensures asymptotic optimality, it does not tell how this parameter should be selected in practice. To answer this question, the authors of [21] establish that the root of the following non linear equation, which we denote here by $\tilde{\theta}$

$$
\mathbb{E}_{g_{\tilde{\theta}}}\left[\sum_{i=0}^{N} \exp \left(Z_{i}\right)\right]=\sum_{i=0}^{N} \exp \left(\mu_{i}+\frac{\Sigma_{i i}}{2(1-\tilde{\theta})}\right)=1 / \gamma_{t h} .
$$

satisfy (19) and hence guarantees the asymptotic optimality of the resulting estimator.

In order to avoid the high complexity required to solve the previous non-linear equation, we propose in the sequel a more simplified approach that directly minimizes the asymptotic variance 
or equivalently the second moment of the IS estimator. In fact, it was proved in [21] that the second moment of $T_{\gamma_{t h}, C M S, \theta}(\mathbf{Z})$ satisfies when $\theta \rightarrow 1$ as $\gamma_{t h} \rightarrow 0$ :

$$
\begin{aligned}
\mathbb{E}_{g_{\theta}}\left[T_{\gamma_{t h}, C M S, \theta}^{2}(\mathbf{Z})\right] & =\Theta\left(\frac{\left(1-\theta^{2}\right)^{-(N+1) / 2}}{\log \left(1 / \gamma_{t h}\right)}\right. \\
& \left.\times \exp \left(-\frac{\left(\log \left(1 / \gamma_{t h}\right)-\mu\right)^{2}(1+\theta)}{2 \sigma^{2}}\right)\right)
\end{aligned}
$$

where $\sigma^{2}=\max _{k} \Sigma_{\mathbf{k k}}, \mu=\max _{k ; \Sigma_{k k}=\sigma^{2}} \mu_{k}$, and $f=\Theta(g)$ means that $f=\mathcal{O}(g)$ and $g=\mathcal{O}(f)$. Thus, we select $\hat{\theta}$ to be the value that minimizes the previous right hand side term. Through a simple computation, a closed-form expression of the minimizer is obtained as follows

$$
\hat{\theta}=\frac{-1+\sqrt{1+4 c^{2}}}{2 c}
$$

where $c=\left(\log \left(1 / \gamma_{t h}\right)-\mu\right)^{2} / 2(N+1) \sigma^{2}$. It is worth noticing that this choice also guarantees the asymptotic optimality property. As a matter of fact, the value of $\hat{\theta}$ satisfies the following relation

$$
(1-\hat{\theta})^{-1} \underset{\gamma_{t h} \rightarrow 0}{\sim} \frac{\left(\log \left(1 / \gamma_{t h}\right)\right)^{2}}{\sigma^{2}(N+1)}
$$

and hence (19) is satisfied.

In the following theorem, we provide asymptotic equivalents for the squared coefficient of variation $^{2}$ associated with the estimators $T_{\gamma_{t h}, C M S, \tilde{\theta}}(\mathbf{Z})$ and $T_{\gamma_{t h}, C M S, \hat{\theta}}(\mathbf{Z})$.

Theorem 1. The estimators $T_{\gamma_{t h}, C M S, \tilde{\theta}}(\mathbf{Z})$ and $T_{\gamma_{t h}, C M S, \hat{\theta}}(\mathbf{Z})$ satisfy respectively:

$$
\frac{\operatorname{var}_{g_{\tilde{\theta}}}\left[T_{\gamma_{t h}, C M S, \tilde{\theta}}(\mathbf{Z})\right]}{\alpha^{2}}=\Theta\left(\left(\frac{1}{\gamma_{t h}}\right)^{\frac{1}{4}} \log \left(\frac{1}{\gamma_{t h}}\right)^{\frac{N+1}{2}+1}\right)
$$

and

$$
\frac{\operatorname{var}_{g_{\hat{\theta}}}\left[T_{\gamma_{t h}, C M S, \hat{\theta}}(\mathbf{Z})\right]}{\alpha^{2}}=\Theta\left(\log \left(\frac{1}{\gamma_{t h}}\right)^{N+2}\right) .
$$

\section{Proof: See Appendix B.}

Theorem 1 provides the coefficient of variation associated with both settings of $\theta$. As will be elaborated on later, this could serve to indicate the order of the number simulation runs needed

\footnotetext{
${ }^{2}$ The coefficient of variation is sometimes called relative error in some references related to rare event simulations, see for instance [22].
} 
by the estimator to ensure a fixed accuracy requirement. In fact, it suffices to select a number of samples that is proportional to the squared coefficient of variation. In particular, the number of simulation runs should be proportional to $\left(\frac{1}{\gamma_{t h}}\right)^{\frac{1}{4}} \log \left(\frac{1}{\gamma_{t h}}\right)^{\frac{N+1}{2}+1}$ when $\theta=\tilde{\theta}$ and to $\log \left(\frac{1}{\gamma_{t h}}\right)^{N+2}$ when $\theta=\hat{\theta}$. Recalling that the naive MC requires a number of simulation runs of the order of $\log \left(\frac{1}{\gamma_{t h}}\right) \exp \left(\frac{\left(\log \left(\frac{1}{\gamma_{t h}}\right)-\mu\right)^{2}}{2 \sigma^{2}}\right)$. Thus, we clearly expect the CMS approach using both settings of $\theta$ to yield a substantial computational gain over naive MC simulations.

For the reader convenience, a pseudo-code describing all steps to estimate $\alpha$ via the proposed CMS IS-based approach is as follows:

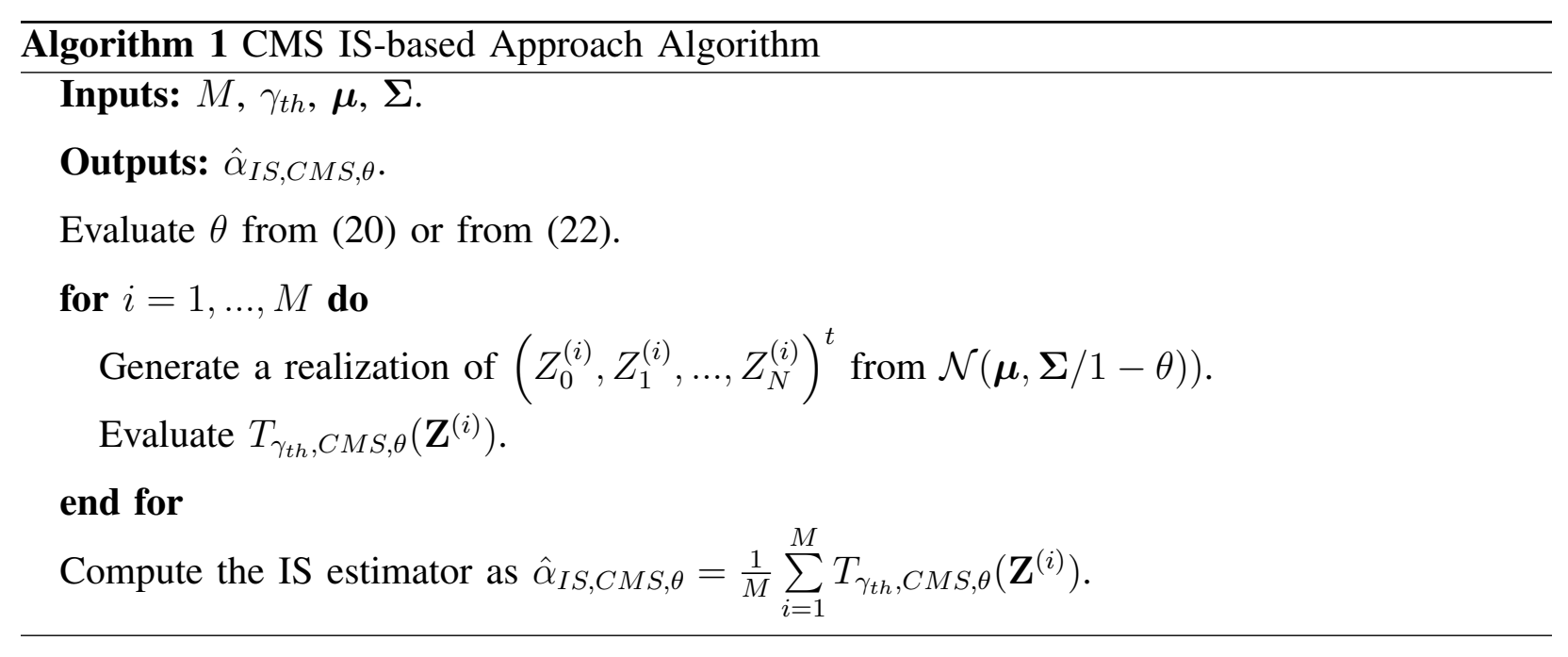

\section{B. MS-CMS IS Approach}

This part is devoted to the analysis of the MS-CMS approach wherein the shifting and the scaling parameter are related as $\theta_{1}=\frac{1}{1-\theta_{2}}$ and $\theta_{2}=\theta$ with $0 \leq \theta<1$. The reason behind using such a particular setting is to simplify the analysis and hence the asymptotic optimality property. The IS PDF corresponding to this scheme is $\mathcal{N}\left(\boldsymbol{\mu}+\frac{a}{1-\theta}, \frac{\boldsymbol{\Sigma}}{(1-\theta)}\right)$ which is given in (15) and will be denoted again by $g_{\theta}(\cdot)$. Recall also that the value of $\theta$ should be chosen such that it goes to 1 as $\gamma_{t h}$ goes to 0. From (16), the likelihood ratio following this MS-CMS IS approach is given 
as follows:

$$
\begin{aligned}
& L\left(Z_{0}, \ldots, Z_{N}\right) \\
& =\frac{\exp \left(-\frac{\theta}{2}(\mathbf{Z}-\boldsymbol{\mu})^{t} \boldsymbol{\Sigma}^{-1}(\mathbf{Z}-\boldsymbol{\mu})-(\mathbf{Z}-\boldsymbol{\mu})^{t} \boldsymbol{\Sigma}^{-1} \boldsymbol{a}\right)}{(1-\theta)^{\frac{N+1}{2}}} \\
& \times \exp \left(\frac{\boldsymbol{a}^{t} \boldsymbol{\Sigma}^{-1} \boldsymbol{a}}{2(1-\theta)}\right) .
\end{aligned}
$$

In the MS-CMS scheme, the RV $T_{\gamma_{t h}}(\mathbf{Z})$ is denoted by $T_{\gamma_{t h}, M S-C M S, \theta}$ and its corresponding IS estimator is denoted by $\hat{\alpha}_{I S, M S-C M S, \theta}$. Applying Lemma 1, we can derive an asymptotic expression of the second moment of the $\mathrm{RV} T_{\gamma_{t h}, M S-C M S, \theta}(\mathbf{Z})$. We obtain the following result: Proposition 1. Assume that $\theta$ is selected such that $\theta \rightarrow 1$ as $\gamma_{t h} \rightarrow 0$. Then, the second moment of $T_{\gamma_{t h}, M S-C M S, \theta}(\mathbf{Z})$ satisfies the following as $\gamma_{t h} \rightarrow 0$ :

$$
\begin{aligned}
& \mathbb{E}_{g_{\theta}}\left[T_{\gamma_{t h}, M S-C M S, \theta}^{2}(\mathbf{Z})\right] \\
& =\Theta\left(\frac{\exp \left(\frac{\boldsymbol{a}^{t} \boldsymbol{\Sigma}^{-1} \boldsymbol{a}}{1-\theta^{2}}-\frac{\left(\log \left(\frac{1}{\gamma_{t h}}\right)+\frac{1}{1+\theta}-\mu\right)^{2}(1+\theta)}{2 \sigma^{2}}\right)}{\log \left(\frac{1}{\gamma_{t h}}\right)\left(1-\theta^{2}\right)^{\frac{N+1}{2}}}\right) .
\end{aligned}
$$

Proof: See Appendix C.

Selecting $\theta$ to be the minimizer of the right-hand side of (27) does not lead to a compact closedform expression for $\theta$ and, furthermore, cannot be easily exploited to establish the asymptotic optimality criterion. On the other hand, one can easily convince oneself that when $\theta$ satisfies $(1-\theta)^{-1}=o\left(\log \left(1 / \gamma_{t h}\right)^{2}\right)$, the asymptotic optimality criterion (12) holds. A plausible choice of $\theta$ can be thus given by:

$$
\bar{\theta}=1-\frac{\sigma^{2}}{2\left(\log \left(\frac{1}{\gamma_{t h}}\right)-\mu\right)} .
$$

Plugging this value into the result of Proposition 1, we prove that the squared coefficient of variation of the MS-CMS estimator is given by:

Theorem 2. The squared coefficient of variation of the estimator $T_{\gamma_{t h}, M S-C M S, \bar{\theta}}(\mathbf{Z})$ is given as follows:

$$
\begin{aligned}
& \frac{\operatorname{var}_{g_{\bar{\theta}}}\left[T_{\gamma_{t h}, M S-C M S, \bar{\theta}}(\mathbf{Z})\right]}{\alpha^{2}} \\
& =\Theta\left(\log \left(\frac{1}{\gamma_{t h}}\right)^{\frac{N+1}{2}+1}\left(\frac{1}{\gamma_{t h}}\right)^{\frac{1}{4}+\frac{a^{t} \boldsymbol{\Sigma}^{-1} \boldsymbol{a}-1}{\sigma^{2}}}\right) .
\end{aligned}
$$


Moreover, the asymptotic optimality criterion (12) holds.

Proof: See Appendix D

As it was mentioned in the previous subsection, the coefficient of variation helps to select the number of required simulation runs in order to meet a fixed accuracy requirement. In fact, Theorem 2 indicates that the number of simulation runs needed to meet a fixed accuracy could be chosen to be proportional to $\log \left(\frac{1}{\gamma_{t h}}\right)^{\frac{N+1}{2}+1}\left(\frac{1}{\gamma_{t h}}\right)^{\frac{1}{4}+\frac{a^{t} \boldsymbol{\Sigma}^{-1} \boldsymbol{a}-1}{\sigma^{2}}}$. Thus, compared to naive MC simulations, it is clearly obvious that the MS-CMS IS will achieve a substantial gain over naive MC simulation in terms of the number of samples that are needed for a fixed accuracy requirement.

For the reader convenience, a pseudo code of the MS-CMS IS approach is provided:

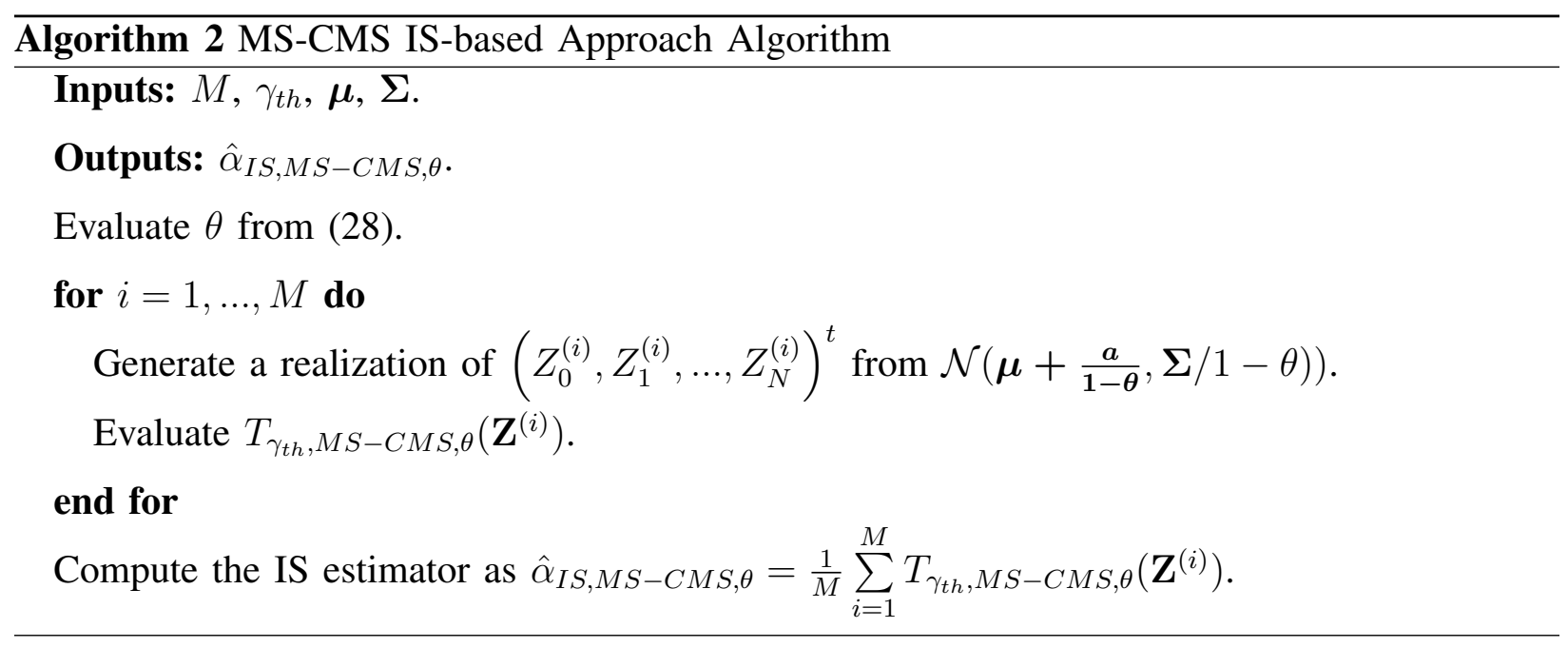

\section{Comparison}

Theorem 1 and Theorem 2 clearly show that asymptotically the CMS approach with $\theta=\hat{\theta}$ should outperform, for very low values of $\gamma_{t h}$, the CMS approach with $\theta=\tilde{\theta}$ as well as the MS-CMS approach. This is because, as $\gamma_{t h} \rightarrow 0$, the coefficient of variation associated with the CMS IS-based approach with $\theta=\hat{\theta}$ increases at a lower rate than that of the CMS approach with $\theta=\tilde{\theta}$ and the MS-CMS IS approach. However, it might happen that for moderately low values of $\gamma_{t h}$, the MS-CMS approach outperforms both CMS based techniques. Given that we are interested in values of $\alpha$ that cover the operating range of OP values, say between $10^{-8}$ and $10^{-3}$, it important, from an engineering perspective, to identify scenarios in which the MS-CMS 
approach may overcome the two versions of the CMS approach and vice versa. A close look at Theorem 1 and Theorem 2 reveals that the sign of $\frac{a^{t} \Sigma^{-1} a-1}{\sigma^{2}}$ and how much it is close to $-\frac{1}{4}$ play a central role in performing this comparison. In fact, in the presence of noise, the quantity $\frac{\boldsymbol{a}^{t} \boldsymbol{\Sigma}^{-1} \boldsymbol{a}-1}{\sigma^{2}}$ appearing in Theorem 2 is equal to $\frac{1 / \sigma_{0}^{2}-1}{\sigma_{0}^{2}+\max _{1 \leq i \leq N} \sigma_{i}^{2}}$. Hence, considering a fixed value of $\max _{1 \leq i \leq N} \sigma_{i}^{2}$, it turns out that $\sigma_{0}^{2}$ is the key parameter for comparing both proposed IS schemes. For the sake of illustration, we plot in Fig. 1 the quantity $\frac{\boldsymbol{a}^{t} \boldsymbol{\Sigma}^{-1} \boldsymbol{a}-1}{\sigma_{0}^{2}+\max _{1 \leq i \leq N} \sigma_{i}^{2}}$ as a function of $\sigma_{0}$ and for a fixed $\max _{1 \leq i \leq N} \sigma_{i}=4 \mathrm{~dB}$. We point out from this figure that, for a small value of $\sigma_{0}$, i.e. $\sigma_{0}<1$ (equivalently $4.345 \mathrm{~dB}$ ), both versions of the CMS approach will certainly outperform the MS-CMS scheme. This is because the term $\frac{\boldsymbol{a}^{t} \boldsymbol{\Sigma}^{-1} \boldsymbol{a}-1}{\sigma_{0}^{2}+\max _{1 \leq i \leq N} \sigma_{i}^{2}}$ is positive in this case. However, as we increase $\sigma_{0}$, which is equivalent to increasing the fluctuations of the desired user power, the term $\frac{\boldsymbol{a}^{t} \boldsymbol{\Sigma}^{-1} \boldsymbol{a}-1}{\sigma^{2}}$ becomes negative. In this situation, we expect the MS-CMS approach to outperform the CMS one. Furthermore, we expect that the higher gain of the MS-CMS approach would be obtained when $\sigma_{0}$ becomes close to the value minimizing $\frac{\boldsymbol{a}^{t} \boldsymbol{\Sigma}^{-1} \boldsymbol{a}-1}{\sigma^{2}}$. In the next section, all the

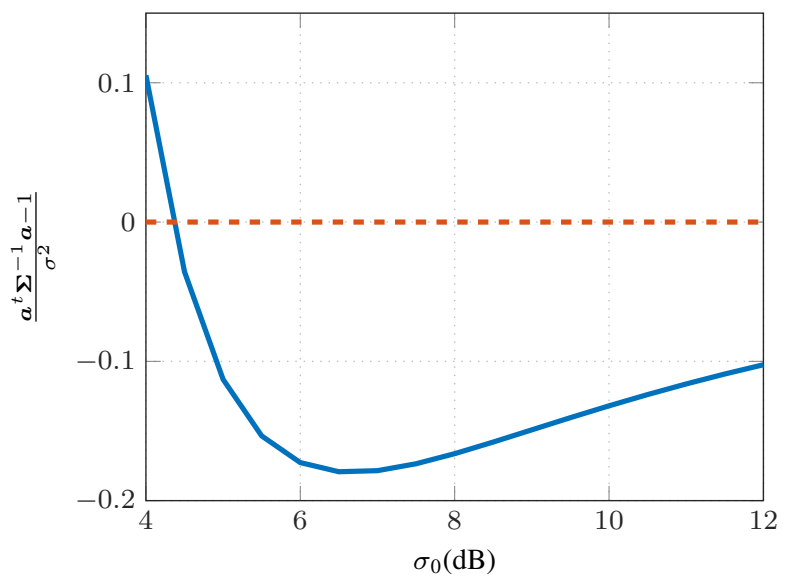

Fig. 1. $\frac{\boldsymbol{a}^{t} \boldsymbol{\Sigma}^{-1} \boldsymbol{a}-1}{\sigma_{0}^{2}+\max _{1 \leq i \leq N} \sigma_{i}^{2}}$ function of $\sigma_{0}$ with $\max _{1 \leq i \leq N} \sigma_{i}=4 \mathrm{~dB}$.

above statements will be validated through various simulation results.

\section{Simulation Results}

In this section, we compare by simulations the performances of the naive MC approach with the CMS-based approaches using the two proposed scaling parameters $\tilde{\theta}$ and $\hat{\theta}$ and the MS-CMS IS-based approach. Particularly, we first assess the accuracy of these methods in 
evaluating the OP of the SINR in a Log-normal environment. Then, we quantify and compare their computational efficiency in terms of the number of simulation runs needed to achieve a given accuracy requirement. This allows us to identify the region over which the CMS approaches outperform the MS-CMS and vice versa. Before introducing our results, we shall first recall for the reader convenience some performance metrics used to gauge the efficiency of the proposed methods.

From the use of the central limit theorem, we define the relative error of naive MC estimator as the relative half-width confidence interval of the estimator

$$
\epsilon_{M C}=C \frac{\sqrt{\alpha(1-\alpha)}}{\sqrt{M_{M C}} \alpha}
$$

where $C$ is the confidence constant (in our simulations, it is chosen to be equal to 1.96 which corresponds to $95 \%$ confidence interval), whereas $M_{M C}$ is the number of simulation runs used in the naive MC simulations. Similarly, the relative errors of the CMS IS-based approach with $\theta=\tilde{\theta}$ and $\theta=\hat{\theta}$ are respectively:

$$
\epsilon_{C M S, \tilde{\theta}}=C \frac{\sqrt{\operatorname{var}_{g_{\tilde{\theta}}}\left[T_{\gamma_{t h}, C M S, \tilde{\theta}}(\mathbf{Z})\right]}}{\sqrt{M_{C M S, \tilde{\theta}}} \alpha}
$$

and

$$
\epsilon_{C M S, \hat{\theta}}=C \frac{\sqrt{\operatorname{var}_{g_{\hat{\theta}}\left[T_{\gamma_{t h}, C M S, \hat{\theta}}(\mathbf{Z})\right]}}}{\sqrt{M_{C M S, \hat{\theta}}} \alpha}
$$

where $M_{C M S, \tilde{\theta}}$ and $M_{C M S, \hat{\theta}}$ denote their corresponding number of simulation runs. Finally, we define the relative error given by the MS-CMS IS-based approach as follows:

$$
\epsilon_{M S-C M S, \bar{\theta}}=C \frac{\sqrt{\operatorname{var}_{g_{\bar{\theta}}}\left[T_{\gamma_{t h}, M S-C M S, \bar{\theta}}(\mathbf{Z})\right]}}{\sqrt{M_{M S-C M S, \bar{\theta}}} \alpha}
$$

where $M_{M S-C M S, \bar{\theta}}$ is the number of simulation runs used by the MS-CMS approach.

In a first experiment, we consider the estimation of the OP of the SINR using $N$ co-channel interferers. The parameters of the desired user power are $\mu_{0}=10 \mathrm{~dB}$ and $\sigma_{0}=4 \mathrm{~dB}$. The cochannel interferers' power are independent and identically distributed variates with parameters $\mu_{i}=0 \mathrm{~dB}$ and $\sigma_{i}=4 \mathrm{~dB}, i=1, \ldots, N$. Fig. 2 reports the OP with respect of the threshold $\gamma_{t h}$ when the number of co-channel interferers takes the values $N=2$ (solid line) and $N=4$ (dashed line). It is worth mentioning that the range of the threshold values used in all the below simulation 


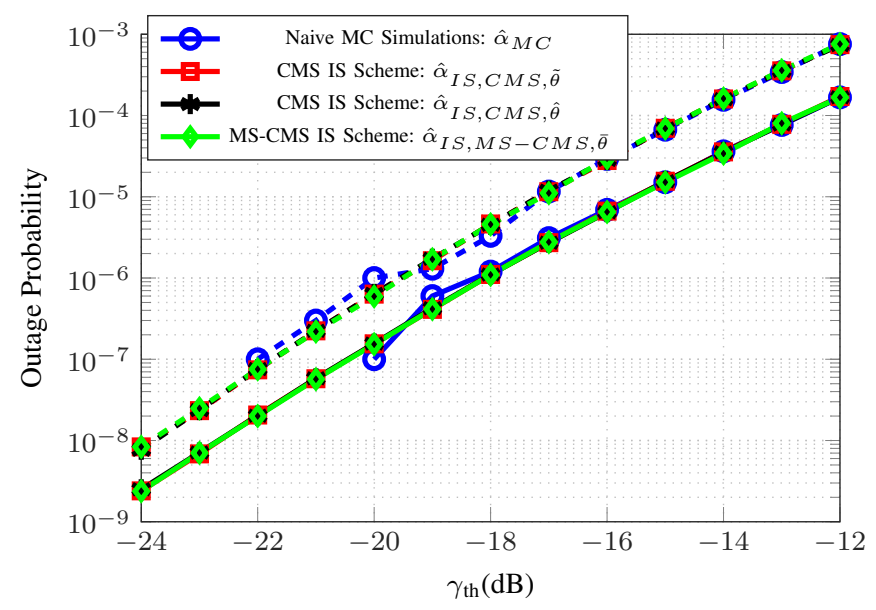

Fig. 2. Outage Probability with $N$ co-channel interferers with parameters : $\mu_{0}=10 \mathrm{~dB}, \sigma_{0}=4 \mathrm{~dB} . N=2$ (Solid Line): $\mu_{i}=0 \mathrm{~dB}$, and $\sigma_{i}=4 \mathrm{~dB}, i=1,2 . N=4$ (Dashed Line): $\mu_{i}=0 \mathrm{~dB}$, and $\sigma_{i}=4 \mathrm{~dB}, i=1,2,3,4 . \eta=-10 \mathrm{~dB}$, $M_{M C}=10^{7}$, and $M_{C M S, \tilde{\theta}}=M_{C M S, \hat{\theta}}=M_{M S-C M S, \bar{\theta}}=5 \times 10^{5}$.

results is chosen such that OP covers the operating range of OP values, say between $10^{-8}$ and $10^{-3}$. The number of simulation runs used by naive MC simulations is $M_{M C}=10^{7}$ whereas $M_{C M S, \tilde{\theta}}=M_{C M S, \hat{\theta}}=M_{M S-C M S, \bar{\theta}}=5 \times 10^{5}$ samples are employed for the three proposed IS schemes. From this figure, we easily point out the limitation of naive MC simulations. In fact, for both choices of $N$, we observe that it ensures a good accuracy as long as the OP is greater than $10^{-5}$. Then, as we decrease the value of $\gamma_{t h}$, it either exhibit an oscillatory behavior or lead to erroneous estimates of the OP equal to zero. This shows that more than $10^{7}$ would be required by naive MC simulations to read an acceptable level of accuracies for very small OP. On the other hand, the two proposed CMS approaches as well as the MS-CMS scheme yield very accurate results using only $5 \times 10^{5}$ simulation runs. This goes in favor towards the performance of the three IS approaches compared to naive MC simulations. We further our analysis by studying the efficiency of the three proposed IS schemes with respect to naive MC simulations in terms of the number of samples needed to ensure a fixed accuracy requirement. To this end, we set an accuracy requirement $\epsilon_{M C}=\epsilon_{C M S, \tilde{\theta}}=\epsilon_{C M S, \hat{\theta}}=\epsilon_{M S-C M S, \bar{\theta}}=5 \%$, and we plot in Fig. 3 the number of samples $M_{M C}, M_{C M S, \tilde{\theta}}, M_{C M S, \hat{\theta}}$, and $M_{M S-C M S, \bar{\theta}}$ needed to ensure this accuracy level as a function of $\gamma_{t h}(N=2$ with solid line and $N=4$ with dashed line). This figure reveals that, for both values of $N$, the proposed IS schemes save a 


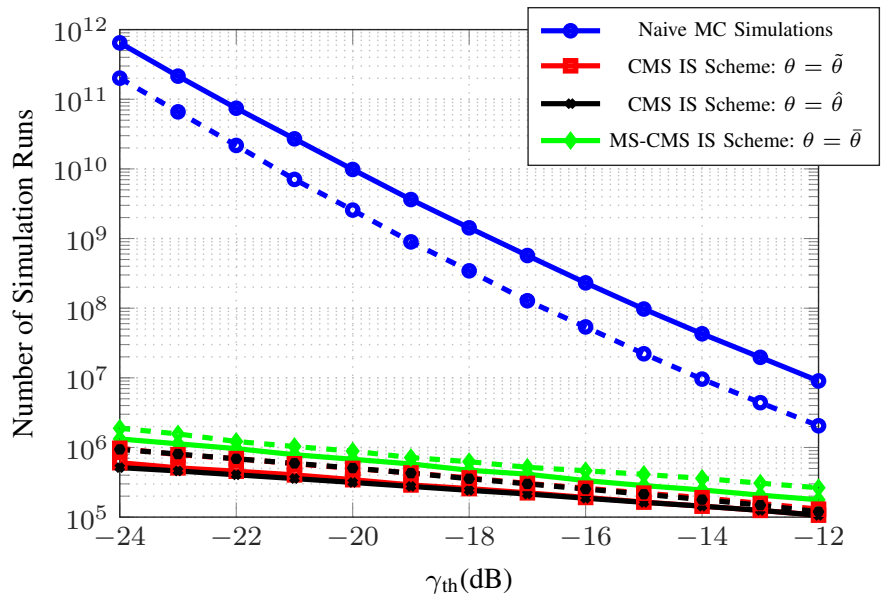

Fig. 3. Number of required simulation runs for $5 \%$ relative error with parameters : $\mu_{0}=10 \mathrm{~dB}, \sigma_{0}=4 \mathrm{~dB}$. $N=2$ (Solid Line): $\mu_{i}=0 \mathrm{~dB}$, and $\sigma_{i}=4 \mathrm{~dB}, i=1,2 . N=4$ (Dashed Line): $\mu_{i}=0 \mathrm{~dB}$, and $\sigma_{i}=4 \mathrm{~dB}, i=1,2,3,4 . \eta=-10 \mathrm{~dB}$.

substantial number of samples compared to naive MC simulation while ensuring the same level of accuracy. Particularly, it is clear from this figure that the number of samples required by naive MC simulations increases at an exponential rate as we decrease the threshold $\gamma_{t h}$, whereas the proposed IS approaches require the number of samples to increase at a slower rate. More precisely, the efficiency of the proposed IS approaches is increasing as we decrease the threshold. This is illustrated in Fig. 3 where, for $95 \%$ accuracy and $N=4$, the naive MC simulation requires approximately $2 \times 10^{6}$ (respectively $2 \times 10^{11}$ ) samples, whereas the two versions of the CMS IS approach need approximately $1.3 \times 10^{5}$ (respectively $10^{6}$ ) simulation runs when $\gamma_{t h}=-12 \mathrm{~dB}$ (respectively $\gamma_{t h}=-24 \mathrm{~dB}$ ).

Fig. 3 reveals also that the CMS IS schemes with $\theta=\tilde{\theta}$ and $\theta=\hat{\theta}$ achieve approximately the same computational efficiency. This in particular suggests working with the closed-form expression of $\hat{\theta}$ in (22) in lieu of $\tilde{\theta}$ requiring to solve the involved non-linear equation in (20). Furthermore, it is worth mentioning that, in this experiment, both versions of the CMS IS approach outperform the MS-CMS IS scheme. For instance when $N=4$ and in the considered range of $\gamma_{t h}$, the MS-CMS approach requires approximately twice the number of samples needed by the two versions of the CMS approach to meet the same accuracy. This result is quite expected from the analysis of the coefficient of variations in Theorem 1 and Theorem 2 as well as the result shown in Fig. 1. One can easily check that under the considered set of parameters, the 
quantity $\frac{\boldsymbol{a}^{t} \boldsymbol{\Sigma}^{-1} \boldsymbol{a}-1}{\sigma^{2}}$ appearing in Theorem 2 is positive and equal approximately to 0.1 . Thus, the coefficient of variation of the MS-CMS approach is worse than the one given by the CMS IS approach with $\theta=\tilde{\theta}$.

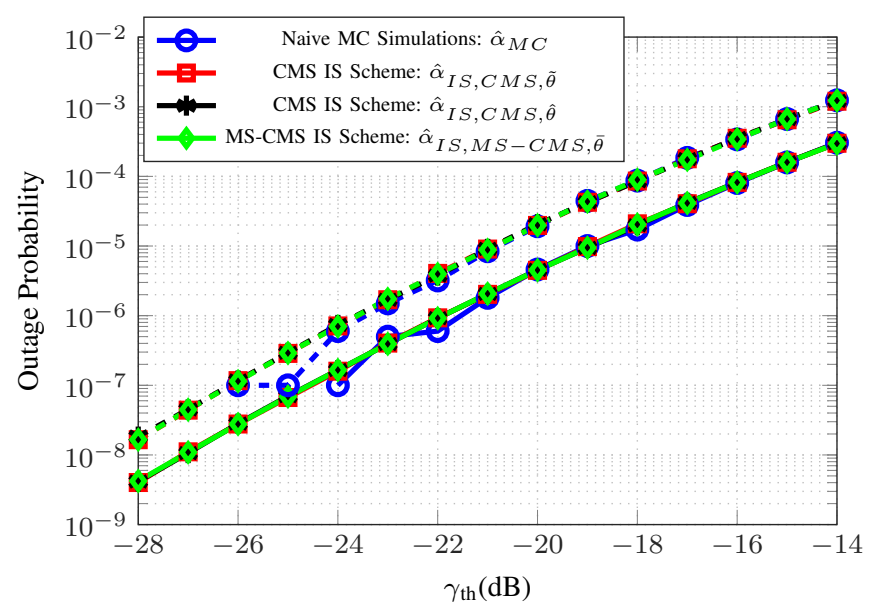

Fig. 4. Outage Probability with $N$ co-channel interferers with parameters : $\mu_{0}=10 \mathrm{~dB}, \sigma_{0}=5 \mathrm{~dB} . N=2$ (Solid Line): $\mu_{i}=0 \mathrm{~dB}$, and $\sigma_{i}=4 \mathrm{~dB}, i=1,2 . N=4$ (Dashed Line): $\mu_{i}=0 \mathrm{~dB}$, and $\sigma_{i}=4 \mathrm{~dB}, i=1,2,3,4 . \eta=-10 \mathrm{~dB}$, $M_{M C}=10^{7}$, and $M_{C M S, \tilde{\theta}}=M_{C M S, \hat{\theta}}=M_{M S-C M S, \bar{\theta}}=5 \times 10^{5}$.

As illustrated in Fig. 1, the MS-CMS approach can outperform the CMS approaches for sufficiently high values of $\sigma_{0}$. To validate this claim, we consider in a second experiment the same set of parameters as before but with $\sigma_{0}=5 \mathrm{~dB}$. Fig. 4 reports the $\mathrm{OP}$ as a function of the threshold $\gamma_{t h}$ for different number of co-channel interferers $(N=2$ with solid line and $N=4$ with dashed line). Similarly to the previous experiment, the three proposed IS schemes yield very accurate results compared to naive MC simulation using less number of samples. In Fig. 5 , we quantify the number of simulation runs needed by the three proposed IS approaches as well as the naive MC simulation to meet a fixed accuracy requirement. Again, the efficiency of the proposed IS schemes over naive MC simulations is clearly established. As seen, when $N=4$ and in order to meet $5 \%$ relative error, about $1.3 \times 10^{6}$ (respectively $10^{11}$ ) samples are needed by naive MC simulation, whereas only about $8.5 \times 10^{4}$ (respectively $3 \times 10^{5}$ ) simulation runs are required by the MS-CMS IS scheme when $\gamma_{t h}=-14 \mathrm{~dB}$ (respectively $\gamma_{t h}=-28 \mathrm{~dB}$ ). Moreover, contrary to the previous results shown in Fig. 3, we note that the MS-CMS approach is more efficient requiring less simulation runs than the two versions of the CMS approach. When 


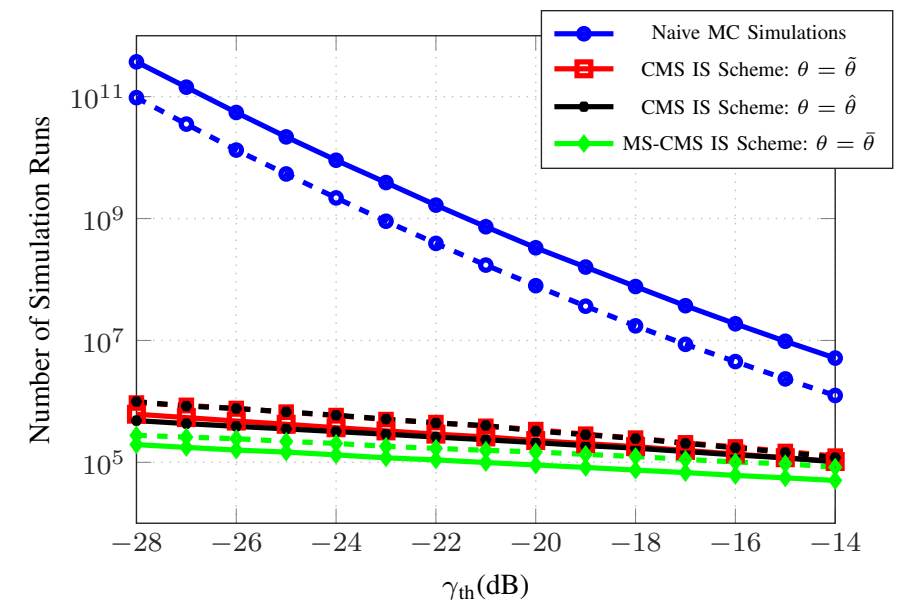

Fig. 5. Number of required simulation runs for $5 \%$ relative error with parameters : $\mu_{0}=10 \mathrm{~dB}, \sigma_{0}=5 \mathrm{~dB} . N=2$ (Solid Line): $\mu_{i}=0 \mathrm{~dB}$, and $\sigma_{i}=4 \mathrm{~dB}, i=1,2 . N=4$ (Dashed Line): $\mu_{i}=0 \mathrm{~dB}$, and $\sigma_{i}=4 \mathrm{~dB}, i=1,2,3,4 . \eta=-10 \mathrm{~dB}$.

$N=4$, the MS-CMS approach requires three times (respectively 1.5 times) less simulation runs than the two version of the CMS approaches when $\gamma_{t h}=-28 \mathrm{~dB}$ (respectively $\gamma_{t h}=-14 \mathrm{~dB}$ ). As it was already mentioned, this result is expected since the quantity $\frac{\boldsymbol{a}^{t} \boldsymbol{\Sigma}^{-1} \boldsymbol{a}-1}{\sigma^{2}}$ is equal in this case to -0.11 approximately, and thus the coefficient of variation of the MS-CMS technique is approximately $\Theta\left(\log \left(\frac{1}{\gamma_{t h}}\right)^{\frac{N+1}{2}+1}\left(\frac{1}{\gamma_{t h}}\right)^{0.14}\right)$, whereas the one given by the CMS approach with $\theta=\tilde{\theta}$ is $\Theta\left(\log \left(\frac{1}{\gamma_{t h}}\right)^{\frac{N+1}{2}+1}\left(\frac{1}{\gamma_{t h}}\right)^{0.25}\right)$.

To further investigate the role played by the term $\frac{a^{t} \boldsymbol{\Sigma}^{-1} \boldsymbol{a}-1}{\sigma^{2}}$ in the efficiency of the MSCMS approach, we further increase $\sigma_{0}$ and set it to $6 \mathrm{~dB}$. With this set of parameters, the quantity $\frac{\boldsymbol{a}^{t} \boldsymbol{\Sigma}^{-1} \boldsymbol{a}-1}{\sigma^{2}}$ is approximately -0.17 and hence the coefficient of variation of the MSCMS approach is $\Theta\left(\log \left(\frac{1}{\gamma_{t h}}\right)^{\frac{N+1}{2}+1}\left(\frac{1}{\gamma_{t h}}\right)^{0.08}\right)$. We thus expect the MS-CMS approach to exhibit a higher gain than the CMS based techniques. We plot again in Fig. 6 the OP as a function of $\gamma_{t h}$. The same conclusions are drawn as in the first and the second experiments. The high accuracy of the proposed IS approaches compared to naive MC simulations is clearly evident using less number of simulation runs. In Fig. 7, we compare the performance of the IS schemes and naive MC simulation in terms of number of samples required to meet a fixed accuracy level. Again all of the proposed IS approaches results in a substantial computational gain over naive MC simulations and the gain is increasing as we decrease the threshold $\gamma_{t h}$. For instance, for $N=4$, the MS-CMS approach requires approximately 16 (respectively $2 \times 10^{5}$ ) times less simulation 


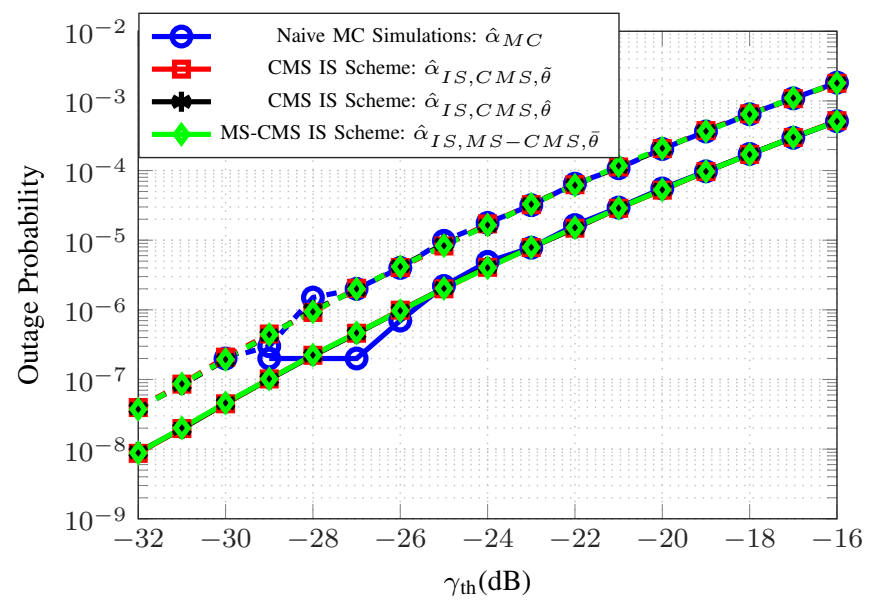

Fig. 6. Outage Probability with $N$ co-channel interferers with parameters : $\mu_{0}=10 \mathrm{~dB}, \sigma_{0}=6 \mathrm{~dB} . N=2$ (Solid Line): $\mu_{i}=0 \mathrm{~dB}$, and $\sigma_{i}=4 \mathrm{~dB}, i=1,2 . N=4$ (Dashed Line): $\mu_{i}=0 \mathrm{~dB}$, and $\sigma_{i}=4 \mathrm{~dB}, i=1,2,3,4 . \eta=-10 \mathrm{~dB}$, $M_{M C}=10^{7}$, and $M_{C M S, \tilde{\theta}}=M_{C M S, \hat{\theta}}=M_{M S-C M S, \bar{\theta}}=5 \times 10^{5}$.

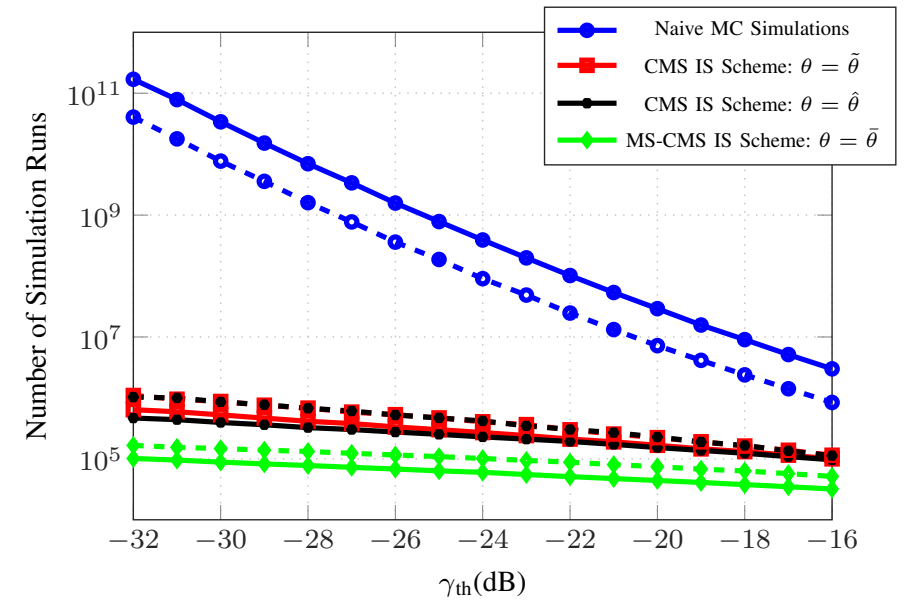

Fig. 7. Number of required simulation runs for $5 \%$ relative error with parameters : $\mu_{0}=10 \mathrm{~dB}, \sigma_{0}=6 \mathrm{~dB} . N=2$ (Solid Line): $\mu_{i}=0 \mathrm{~dB}$, and $\sigma_{i}=4 \mathrm{~dB}, i=1,2 . N=4$ (Dashed Line): $\mu_{i}=0 \mathrm{~dB}$, and $\sigma_{i}=4 \mathrm{~dB}, i=1,2,3,4 . \eta=-10 \mathrm{~dB}$.

runs than the naive MC approach when $\gamma_{t h}=-16 \mathrm{~dB}$ (respectively $\gamma_{t h}=-32 \mathrm{~dB}$ ). Moreover, as expected, the MS-CMS approach outperforms the two version of the CMS methods and the gain is higher than the one in the second experiment. 


\section{CONCLUSION}

In this work, we proposed an efficient hybrid IS approach based on a combination of a mean shifting and a covariance matrix scaling techniques to estimate the OP of the SINR over Lognormal fading channels. Our approach hinges on the observation that the estimation of the OP is equivalent to computing the probability that a sum of correlated Log-normal variates exceeds a given threshold, thereby carrying the considered problem into the standard framework of variance reduction techniques. Two particular cases of the hybrid IS scheme were considered, namely; the CMS and the MS-CMS approaches. We furthered our analysis by providing asymptotic expressions of coefficient of variations given by these two schemes. Moreover, we showed that they achieve the asymptotic optimality property. Furthermore, we identified the regions where the CMS approach outperforms the MS-CMS method and vice versa.

\section{REFERENCES}

[1] N. Ben Rached, A. Kammoun, M.-S. Alouini, and R. Tempone, "An efficient simulation scheme of the outage probability with co-channel interference," in Proc. of the IEEE Global Communications Conference (GLOBECOM,15), San Diego, CA, USA, Dec. 2015.

[2] G. L. Stüber, Principles of Mobile Communication, 2nd Edition. Norwell, MA, USA: Kluwer Academic Publishers, 2001.

[3] M. Ghavami, R. Kohno, and L. Michael, Ultra Wideband Signals and Systems in Communication Engineering. Chichester: Wiley, 2004.

[4] S. M. Navidpour, M. Uysal, and M. Kavehrad, "BER performance of free-space optical transmission with spatial diversity." IEEE Transactions on Wireless Communications, vol. 6, no. 8, pp. 2813-2819, Aug. 2007.

[5] C. Fischione, F. Graziosi, and F. Santucci, "Approximation for a sum of on-off Lognormal processes with wireless applications," IEEE Transactions on Communications, vol. 55, no. 10, pp. 1984-1993, Oct. 2007.

[6] L. Fenton, "The sum of Log-normal probability distributions in scatter transmission systems." IRE Transactions on Communications Systems, vol. 8, no. 1, pp. 57-67, 1960.

[7] M. Pratesi, F. Santucci, and F. Graziosi, "Generalized moment matching for the linear combination of Lognormal RVs - Application to outage analysis in wireless systems," in Proc. of the IEEE Personal, Indoor and Mobile Radio Communications (PIMRC'2006), Sept. 2006.

[8] S. C. Schwartz and Y. S. Yeh, "On the distribution function and moments of power sums with Lognormal component," The Bell Systems Technical Journal, 1982.

[9] N. Mehta, J. Wu, A. Molisch, and J. Zhang, "Approximating a sum of random variables with a Lognormal," IEEE Transactions on Wireless Communications, vol. 6, no. 7, pp. 2690-2699, Jul. 2007.

[10] M. Di Renzo, F. Graziosi, and F. Santucci, "Approximating the linear combination of Log-normal RVs via Pearson type IV distribution for UWB performance analysis," IEEE Transactions on Communications, vol. 57, no. 2, pp. 388-403, Feb. 2009. 
[11] S. Chen, H. Nie, and B. Ayers-Glassey, "Lognormal sum approximation with a variant of type IV Pearson distribution," IEEE Communications Letters, vol. 12, no. 9, pp. 630-632, Sept. 2008.

[12] M. Di Renzo, F. Graziosi, and F. Santucci, "Further results on the approximation of Log-normal power sum via Pearson type IV distribution: A general formula for log-moments computation,” IEEE Transactions on Communications, vol. 57, no. 4, pp. 893-898, Apr. 2009.

[13] N. C. Beaulieu and F. Rajwani, "Highly accurate simple closed-form approximations to Lognormal sum distributions and densities," IEEE Communications Letters, vol. 8, no. 12, pp. 709-711, Dec. 2004.

[14] N. C. Beaulieu and Q. Xie, “An optimal Lognormal approximation to Lognormal sum distributions," IEEE Transactions on Vehicular Technology, vol. 53, no. 2, pp. 479-489, Mar. 2004.

[15] G. P. Fettweis, “The tactile internet: Applications and challenges,” IEEE Vehicular Technology Magazine, vol. 9, no. 1, pp. 64-70, Mar. 2014.

[16] N. C. Beaulieu, E. Biglieri, and M. Lai, "Sample rejection and importance sampling in the simulation of multidimensional signalling systems," IEE Proceedings I - Communications, Speech and Vision, vol. 140, no. 6, pp. 445-452, Dec. 1993.

[17] P. J. Smith, M. Shafi, and H. Gao, "Quick simulation: a review of importance sampling techniques in communications systems," IEEE Journal on Selected Areas in Communications, vol. 15, no. 4, pp. 597-613, May. 1997.

[18] N. Ben Rached, F. Benkhelifa, M.-S. Alouini, and R. Tempone, "A fast simulation method for the Log-normal sum distribution using a hazard rate twisting technique," in Proc. of the IEEE International Conference on Communications (ICC'15), London, UK, Jun 2015.

[19] N. Ben Rached, A. Kammoun, M.-S. Alouini, and R. Tempone, "An improved hazard rate twisting approach for the statistic of the sum of subexponential variates," IEEE Communications Letters, vol. 19, no. 1, pp. 14-17, Jan. 2015.

[20] _ _ "Unified importance sampling schemes for efficient simulation of outage capacity over generalized fading channels," IEEE Journal of Selected Topics in Signal Processing, vol. 10, no. 2, pp. 376-388, Mar. 2016.

[21] S. Asmussen, J. H. Blanchet, S. Juneja, and L. Rojas-Nandayapa, "Efficient simulation of tail probabilities of sums of correlated Lognormals," Annals OR, vol. 189, no. 1, pp. 5-23, 2011.

[22] S. Asmussen and P. W. Glynn, Stochastic simulation : algorithms and analysis, ser. Stochastic modelling and applied probability. New York: Springer, 2007.

[23] P. Loskot and N. C. Beaulieu, "Sample rejection for efficient simulation of binary coding schemes over quantized additive white gaussian noise channels," IEEE Transactions on Communications, vol. 53, no. 7, pp. 1145-1154, Jul. 2005.

[24] J. A. Bucklew, Introduction to Rare Event Simulation, ser. Springer series in statistics. New York: Springer, 2004.

[25] C.-L. I. and B. B. Lusignan, "The 'optimum' BER estimator for digital satellite communication systems," in 11th Communication Satellite Systems Conference, 1986, pp. 144-151.

[26] N. C. Beaulieu, "An investigation of gaussian tail and rayleigh tail density functions for importance sampling digital communication system simulation,” IEEE Transactions on Communications, vol. 38, no. 9, pp. 1288-1292, Sep. 1990.

[27] A. Gulisashvili and P. Tankov, "Tail behavior of sums and differences of log-normal random variables," Bernoulli, vol. 22, no. 1, pp. 444-493, 02 2016. [Online]. Available: http://dx.doi.org/10.3150/14-BEJ665

[28] S. Asmussen and L. Rojas-Nandayapa, "Asymptotics of sums of lognormal random variables with gaussian copula," Statistics \& Probability Letters, vol. 78, no. 16, pp. 2709-2714, 2008. 


\section{APPENDIX A}

\section{ProOF OF LEMMA 1}

The second moment of $T_{\gamma_{t h}}(\mathbf{Z})$ is:

$$
\begin{aligned}
& \mathbb{E}_{g_{\theta_{1}, \theta_{2}}}\left[T_{\gamma_{t h}}^{2}(\mathbf{Z})\right]=\mathbb{E}_{f}\left[T_{\gamma_{t h}}(\mathbf{Z})\right] \\
& =\int_{\left\{\sum_{i=0}^{N} \exp \left(z_{i}\right)>\frac{1}{\gamma_{t h}}\right\}} L\left(z_{0}, \ldots, z_{N}\right) f\left(z_{0}, \ldots, z_{n}\right) d z_{0} \ldots d z_{N} .
\end{aligned}
$$

Upon putting the expression of the likelihood ratio (16) into (34), we get

$$
\begin{aligned}
& \mathbb{E}_{g_{\theta_{1}, \theta_{2}}}\left[T_{\gamma_{t h}}^{2}(\mathbf{Z})\right] \\
& =\frac{\exp \left(\frac{\left(1-\theta_{2}\right) \theta_{1}^{2}}{2} \boldsymbol{a}^{t} \boldsymbol{\Sigma}^{-1} \boldsymbol{a}+\left(1-\theta_{2}\right) \theta_{1} \boldsymbol{\mu}^{t} \boldsymbol{\Sigma}^{-1} \boldsymbol{a}\right)}{\left(1-\theta_{2}^{2}\right)^{\frac{N+1}{2}} \sqrt{\operatorname{det}(\boldsymbol{\Sigma})(2 \pi)^{N+1}}} \\
& \times \int_{\left\{\sum_{i=0}^{N} \exp \left(z_{i}\right)>\frac{1}{\gamma_{t h}}\right\}} \exp \left(-\frac{1+\theta_{2}}{2}(\boldsymbol{z}-\boldsymbol{\mu})^{t} \boldsymbol{\Sigma}^{-1}(\boldsymbol{z}-\boldsymbol{\mu})\right) \\
& \times \exp \left(-\left(1-\theta_{2}\right) \theta_{1} \boldsymbol{a}^{t} \boldsymbol{\Sigma}^{-1} \boldsymbol{a}\right) d z_{0} \ldots d z_{N} \\
& =\frac{\exp \left(\frac{\left(1-\theta_{2}\right) \theta_{1}^{2}}{2} \boldsymbol{a}^{t} \boldsymbol{\Sigma}^{-1} \boldsymbol{a}+\left(1-\theta_{2}\right) \theta_{1} \boldsymbol{\mu}^{t} \boldsymbol{\Sigma}^{-1} \boldsymbol{a}\right)}{\left(1-\theta_{2}^{2}\right)^{\frac{N+1}{2}} \sqrt{\operatorname{det}(\boldsymbol{\Sigma})(2 \pi)^{N+1}}} \\
& \times \int_{\left\{\sum_{i=0}^{N} \exp \left(z_{i}\right)>\frac{1}{\gamma_{t h}}\right\}} \exp \left(-\frac{1+\theta_{2}}{2}\left(\boldsymbol{z}-\boldsymbol{\mu}_{\mathbf{1}}\right)^{t} \boldsymbol{\Sigma}^{-1}\left(\boldsymbol{z}-\boldsymbol{\mu}_{\mathbf{1}}\right)\right) \\
& \times \exp \left(-\frac{1+\theta_{2}}{2}\left[\boldsymbol{\mu}^{t} \boldsymbol{\Sigma}^{-1} \boldsymbol{\mu}-\boldsymbol{\mu}_{\mathbf{1}}{ }^{t} \boldsymbol{\Sigma}^{-1} \boldsymbol{\mu}_{\mathbf{1}}\right]\right) d z_{0} \ldots d z_{N}
\end{aligned}
$$

where $\boldsymbol{\mu}_{\mathbf{1}}=\boldsymbol{\mu}-\frac{\left(1-\theta_{2}\right) \theta_{1}}{1+\theta_{2}} \boldsymbol{a}$. After a straightforward computation, it follows that:

$$
\begin{aligned}
& \mathbb{E}_{g_{\theta_{1}, \theta_{2}}}\left[T_{\gamma_{t h}}^{2}(\mathbf{Z})\right]=\left(1-\theta_{2}^{2}\right)^{-\frac{N+1}{2}} \exp \left(\frac{\left(1-\theta_{2}\right) \theta_{1}^{2}}{2} \boldsymbol{a}^{t} \boldsymbol{\Sigma}^{-1} \boldsymbol{a}\right) \\
& \times \exp \left(\frac{\left(1-\theta_{2}\right)^{2} \theta_{1}^{2}}{2\left(1+\theta_{2}\right)} \boldsymbol{a}^{t} \boldsymbol{\Sigma}^{-1} \boldsymbol{a}\right) \\
& \times P_{\theta_{1}, \theta_{2}}\left(\sum_{i=0}^{N} \exp \left(Z_{i}\right)>\frac{1}{\gamma_{t h}}\right) \\
& =\left(1-\theta_{2}^{2}\right)^{-\frac{N+1}{2}} \exp \left(\frac{\left(1-\theta_{2}\right) \theta_{1}^{2}}{1+\theta_{2}} \boldsymbol{a}^{t} \boldsymbol{\Sigma}^{-1} \boldsymbol{a}\right) \\
& \times P_{\theta_{1}, \theta_{2}}\left(\sum_{i=0}^{N} \exp \left(Z_{i}\right)>\frac{1}{\gamma_{t h}}\right)
\end{aligned}
$$


where $P_{\theta_{1}, \theta_{2}}(\cdot)$ is the probability measure under which the random vector $\mathbf{Z}$ follows a multivariate normal with mean vector $\boldsymbol{\mu}_{1}$ and covariance matrix $\frac{\boldsymbol{\Sigma}}{1+\theta_{2}}$.

\section{APPENDIX B}

\section{ProOF OF THEOREM 1}

The result in (24) was proven in [21]. As for the second part of the proof, we start by plugging the value of $\hat{\theta}$ in (22) into the asymptotic expression in (21). In doing so, we obtain:

$$
\begin{aligned}
& \mathbb{E}_{g_{\hat{\theta}}}\left[T_{\gamma_{t h}, C M S, \hat{\theta}}^{2}(\mathbf{Z})\right]=\Theta\left(\frac{(1-\hat{\theta})^{-(N+1) / 2}}{\log \left(1 / \gamma_{t h}\right)}\right. \\
& \left.\times \exp \left(-\frac{\left(\log \left(1 / \gamma_{t h}\right)-\mu\right)^{2}(1+\hat{\theta})}{2 \sigma^{2}}\right)\right) .
\end{aligned}
$$

Now, using (23) and the fact that

$$
1+\hat{\theta}=2-\frac{(N+1) \sigma^{2}}{\left(\log \left(\frac{1}{\gamma_{t h}}\right)-\mu\right)^{2}}+o\left(\frac{1}{\left(\log \left(\frac{1}{\gamma_{t h}}\right)-\mu\right)^{2}}\right)
$$

the expression in (37) becomes

$$
\begin{aligned}
\mathbb{E}_{g_{\hat{\theta}}}\left[T_{\gamma_{t h}, C M S, \hat{\theta}}^{2}(\mathbf{Z})\right]= & \Theta\left(\log \left(\frac{1}{\gamma_{t h}}\right)^{N}\right. \\
& \left.\exp \left(-\frac{\left(\log \left(\frac{1}{\gamma_{t h}}\right)-\mu\right)^{2}}{\sigma^{2}}\right)\right) .
\end{aligned}
$$

On the other hand, the probability of interest $\alpha$ satisfies [28]:

$$
\alpha \sim \frac{\sigma}{\sqrt{2 \pi}\left(\log \left(\frac{1}{\gamma_{t h}}\right)-\mu\right)} \exp \left(-\frac{\left(\log \left(\frac{1}{\gamma_{t h}}\right)-\mu\right)^{2}}{2 \sigma^{2}}\right) .
$$

Finally, by combining the previous asymptotic expression together with (39), we conclude the proof. 


\section{APPENDIX C}

\section{PROOF OF PROPOSITION 1}

Replacing $\theta_{2}$ by $\theta$ and $\theta_{1}$ by $\frac{1}{1-\theta_{2}}$ in the result of Lemma 1, it follows that:

$$
\begin{aligned}
& \mathbb{E}_{g_{\theta}}\left[T_{\gamma_{t h}, M S-C M S, \theta}^{2}(\mathbf{Z})\right]=\left(1-\theta^{2}\right)^{-\frac{N+1}{2}} \\
& \times \exp \left(\frac{\boldsymbol{a}^{t} \boldsymbol{\Sigma}^{-1} \boldsymbol{a}}{1-\theta^{2}}\right) P_{\theta}\left(\sum_{i=0}^{N} \exp \left(Z_{i}\right)>\frac{\exp \left(\frac{1}{1+\theta}\right)}{\gamma_{t h}}\right)
\end{aligned}
$$

where $P_{\theta}(\cdot)$ is the probability measure under which the random vector $\mathbf{Z}$ is a multivariate normal with mean vector $\boldsymbol{\mu}$ and covariance matrix $\frac{\boldsymbol{\Sigma}}{1+\theta}$. Given that $\theta \rightarrow 1$ as $\gamma_{t h} \rightarrow 0$, we have, using the result in [21], that

$$
\begin{aligned}
& P_{\theta}\left(\sum_{i=0}^{N} \exp \left(Z_{i}\right)>\frac{\exp \left(\frac{1}{1+\theta}\right)}{\gamma_{t h}}\right) \\
& \sim \frac{s \sigma \exp \left(-\frac{\left(\log \left(\frac{1}{\gamma_{t h}}\right)+\frac{1}{1+\theta}-\mu\right)^{2}(1+\theta)}{2 \sigma^{2}}\right)}{\sqrt{2 \pi(1+\theta)}\left(\log \left(\frac{1}{\gamma_{t h}}\right)+\frac{1}{1+\theta}-\mu\right)} \\
& =\Theta\left(\frac{\exp \left(-\frac{\left(\log \left(\frac{1}{\gamma_{t h}}\right)+\frac{1}{1+\theta}-\mu\right)^{2}(1+\theta)}{2 \sigma^{2}}\right)}{\log \left(\frac{1}{\gamma_{t h}}\right)}\right) .
\end{aligned}
$$

Hence, by combining the previous result in (41), we conclude the proof. 


\section{APPENDIX D}

\section{PROOF OF THEOREM 2}

Using Proposition 1 and the value of $\bar{\theta}$ in (28), we get after straightforward computations that:

$$
\begin{aligned}
& \mathbb{E}_{g_{\bar{\theta}}}\left[T_{\gamma_{t h}, M S-C M S, \bar{\theta}}^{2}(\mathbf{Z})\right]=\Theta\left(\log \left(\frac{1}{\gamma_{t h}}\right)^{\frac{N+1}{2}-1}\left(\frac{1}{\gamma_{t h}}\right)^{\frac{1}{4}}\right. \\
& \times \exp \left(-\frac{\left(\log \left(\frac{1}{\gamma_{t h}}\right)-\mu\right)^{2}}{\sigma^{2}}\right) \\
& \left.\times \exp \left(\left(\boldsymbol{a}^{t} \boldsymbol{\Sigma}^{-1} \boldsymbol{a}-1\right) \frac{4\left(\log \left(\frac{1}{\gamma_{t h}}\right)-\mu\right)^{2}}{4 \sigma^{2}\left(\log \left(\frac{1}{\gamma_{t h}}\right)-\mu\right)-\sigma^{4}}\right)\right) \\
& =\Theta\left(\log \left(\frac{1}{\gamma_{t h}}\right)^{\frac{N+1}{2}-1}\left(\frac{1}{\gamma_{t h}}\right)^{\frac{1}{4}}\right. \\
& \times \exp \left(-\frac{\left(\log \left(\frac{1}{\gamma_{t h}}\right)-\mu\right)^{2}}{\sigma^{2}}\right) \\
& \times \exp \left(\frac{\boldsymbol{a}^{t} \boldsymbol{\Sigma}^{-1} \boldsymbol{a}-1}{\sigma^{2}}\left(\log \left(\frac{1}{\gamma_{t h}}\right)-\mu\right)\right) .
\end{aligned}
$$

Now, using the asymptotic expression in (40), it follows that

$$
\begin{aligned}
& \frac{\operatorname{var}_{g_{\bar{\theta}}}\left[T_{\gamma_{t h}, M S-C M S, \bar{\theta}}(\mathbf{Z})\right]}{\alpha^{2}} \\
= & \Theta\left(\log \left(\frac{1}{\gamma_{t h}}\right)^{\frac{N+1}{2}+1}\left(\frac{1}{\gamma_{t h}}\right)^{\frac{1}{4}+\frac{a^{t} \boldsymbol{\Sigma}^{-1} \boldsymbol{a - 1}}{\sigma^{2}}}\right)
\end{aligned}
$$

which conclude the first part of the proof. It remains thus to establish the asymptotic optimality property. Recall that this property holds if $\frac{\operatorname{var}_{g_{\bar{\theta}}}\left[T_{\gamma_{t h}, M S-C M S, \bar{\theta}}(\mathbf{Z})\right]}{\alpha^{2-\epsilon}} \rightarrow 0$ for all $\epsilon>0$. [20]. To estbalish this, we shall exploit (44) and 40. We thus have for any $\epsilon>0$ :

$$
\begin{aligned}
& \frac{\operatorname{var}_{g_{\bar{\theta}}}\left[T_{\gamma_{t h}, M S-C M S, \bar{\theta}}(\mathbf{Z})\right]}{\alpha^{2-\epsilon}} \\
& =\Theta\left(\log \left(\frac{1}{\gamma_{t h}}\right)^{\frac{N+1}{2}+1-\epsilon}\left(\frac{1}{\gamma_{t h}}\right)^{\frac{1}{4}+\frac{a^{\boldsymbol{t}^{-1}} \boldsymbol{\Sigma}^{a-1}}{\sigma^{2}}}\right. \\
& \left.\times \exp \left(-\frac{\epsilon}{2 \sigma^{2}}\left(\log \left(\frac{1}{\gamma_{t h}}\right)-\mu\right)^{2}\right)\right)
\end{aligned}
$$

which goes to 0 as $\gamma_{t h}$ goes to 0 for all $\epsilon>0$. Hence the MS-CMS IS-based approach estimate $\alpha$ asymptotically optimal. 